BULL. AUSTRAL. MATH. SOC.

VOL. I (1969), 231-235.

\title{
On the lattice of congruences on a regular semigroup
}

\author{
T. E. Hall
}

A result of Reilly and Scheiblich for inverse semigroups is proved true also for regular semigroups. For any regular semigroup $S$ the relation $\theta$ is defined on the lattice, $\Lambda(S)$, of congruences on $S$ by: $(\rho, \tau) \in \theta$ if $\rho$ and $\tau$ induce the same partition of the idempotents of $S$. Then $\theta$ is a congruence on $\Lambda(S)$, $\Lambda(S) / \Theta$ is complete and the natural homomorphism of $\Lambda(S)$ onto $\Lambda(S) / \Theta$ is a complete lattice homomorphism.

\section{Introduction and summary}

Let $S$ be a semigroup, $E$ its set of idempotents, $\Lambda(S)$ its lattice of congruences, and define on $\Lambda(S)$ the relation

$$
\theta=\{(\rho, \sigma) \in \Lambda(S) \times \Lambda(S): \rho \cap(E \times E)=\sigma \cap(E \times E)\} .
$$

Using the work of Munn [4] and Lallement [2], Reilly and Scheiblich [5] have proved the following

THEOREM 3.4 of [5]. If $S$ is a regular semigroup then

(i) $\theta$ is a meet compatible equivalence on $\Lambda(S)$;

(ii) each $\theta$-class is a complete modular sublattice of $\Lambda(S)$.

For inverse semigroups they prove considerably more in Theorem 5.1 [5]. We consider whether or not Theorem 5.1 [5] holds true also for regular semigroups. We answer this in the affirmative by proving the following

Received 21 March 1969. Received by J. Austral. Math. Soc. 6 December 1968. Communicated by G.B. Preston. This research was carried out at Monash University while the author held a Commonwealth Postgraduate Award. The author wishes to thank his supervisor, Professor G.B. Preston, for an inspiring discussion and further invaluable help. 
theorem, which reduces to Theorem 5.1 [5] when $S$ is an inverse semigroup.

MAIN THEOREM. Let $S$ be a regular semigroup, $E$ the set of idempotents of $S$, and $\Lambda(S)$ the Zattice of congruences on $S$. Define on $\Lambda(S)$ the relation

$$
\theta=\{(\rho, \sigma) \in \Lambda(S) \times \Lambda(S): \rho \cap(E \times E)=\sigma \cap(E \times E)\} .
$$

Then

(i) $\Theta$ is a congruence on $\Lambda(S)$;

(ii) each $\Theta$-class is a complete modular sublattice of $\Lambda(S)$;

(iii) the quotient lattice $\Lambda(S) / \theta$ is complete and the natural homomorphism $\theta^{4}$ of $\Lambda(S)$ onto $\Lambda(S) / \theta$ is a complete Zattice homomorphism.

We use wherever possible, and often without comment, the notations and conventions of Clifford and Preston [1]. For any equivalence $E$ on $S$, we shall often denote the equivalence $E \cap(E \times E)$ by $E \mid E$. We shall use the following well-known result, which one may readily verify.

RESULT 1. Let $L$ be a complete lattice and $\Theta$ a congmence on $L$ satisfying the following condition.

(A) If $\left\{a_{i}: i \in I\right\}$ and $\left\{b_{i}: i \in I\right\}$ (I is some index set) are any two subsets of $L$ such that $\left(a_{i}, b_{i}\right) \in \theta$ for each $i \in I$, then $\left(\bigvee_{i \in I} a_{i}, \underset{i \in I}{\bigvee} b_{i}\right) \in \theta$ and $\left(\bigwedge_{i \in I} a_{i}, \bigwedge_{i \in I} b_{i}\right) \in \theta$.

Then the quotient lattice $L / O$ is a complete lattice and the natural homomorphism $\Theta^{4}$ of $L$ onto $L / \theta$ is a complete lattice homomorphism.

\section{2. $K(\sigma)$-regularity}

This section gives a routine generalization of Lallement's Theorem 3.6 [3] (see also Theorem 2.3[2]).

Let $S$ be a semigroup and $\sigma$ any congruence on $S$. Let $K$ be any one of Green's relations $R, L, H, D, J$ on $S$ (see [I]). To indicate that we are considering one of Green's relations on a different semigroup $T$ say, we shall use, for example, the phrase "K on $T$ ". Define now $K(\sigma)=\{(a, b) \in S \times S: a \sigma$ and $b \sigma$ are $K$-related in $S / \sigma\}$. Then clearly 
$K(\sigma)$ is an equivalence relation on $S, K \subseteq K(\sigma)$, and $\sigma \subseteq K(\sigma)$. Also clearly $H(\sigma)=R(\sigma) \cap L(\sigma)$ and $D(\sigma)=R(\sigma) \circ L(\sigma)=L(\sigma) \circ R(\sigma)=L(\sigma) \vee R(\sigma)$.

For any element $a$ in $S$, let $K(\sigma)_{a}\left[K_{\alpha}\right]$ denote the $K(\sigma)$-class [K-class] of $S$ containing the element $a$, and let $K_{a \sigma}$ denote the $K$-class of $S / \sigma$ containing the element $a \sigma$. Clearly there is a one-to-one mapping, $\psi$ say, from the set of $K(\sigma)$-classes of $S$ onto the set of $K$-classes of $S / \sigma$ such that, for any element $a$ in $S, K(\sigma) a_{a} \psi=K_{a \sigma}$.

Now for $K \neq D$, there is a natural ordering $\leq$ (see [1] or [2]) on the set of $K$-classes of any semigroup. We define $\leq$ on the set of $K(\sigma)$-classes of $S$ (for $K \neq 0$ ), as follows. For any two elements $a, b$ in $S, K(\sigma)_{a} \leq K(\sigma)_{b}$ if and only if $K_{\alpha \sigma} \leq K_{b \sigma}$. Since $\psi$ is a one-to-one mapping, $\leq$ is a partial ordering. Note, for example, that $R(\sigma)_{a} \leq R(\sigma)_{b}$ if and only if

$$
\left\{a \sigma^{k}\right\} \cup\left(a \sigma^{h}\right)\left(S \sigma^{4}\right) \subseteq\left\{b \sigma^{h}\right\} \cup\left(b \sigma^{h}\right)\left(S \sigma^{h}\right) \text {. }
$$

For any two elements $a, b$ in $S$, the following are clear.

(i) If $K_{a}=K_{b}$, then $K(\sigma)_{a}=K(\sigma)_{b}$ (since $\left.K \subseteq K(\sigma)\right)$.

(ii) For $K \neq D$, if $K_{a} \leq K_{b}$, then $K(\sigma)_{a} \leq K(\sigma)_{b}$.

DEFINITION (from [2]) Let $E$ be any equivalence relation on $S$. Then $S$ is called E-regular if, for any congruence $\rho$ on $S, \rho \mid E \subseteq E$ implies $\rho \subseteq E$.

THEOREM 1. If $S$ is a regular semigroup, then $S$ is K(o)-regular.

Proof. With minor alterations (involving (i) and (ii) above), Lallement's proof of Theorem 3.6 [3] is sufficient (see also Theorem 2.3 [2]). The alterations consist mainly in replacing, for example, $R_{a}$ by $R(\sigma)_{a}$

\section{The lattice of congruences}

LEMMA 1. Let $S$ be a regular semigroup. For each element $i$ of some index set $I$, let $\rho_{i}, \rho_{i}^{\prime}$ be congruences on $S$ such that 
$\left(\rho_{i}, \rho_{i}^{\prime}\right) \in \theta$. Then

$$
\left(\underset{i \in I}{V} \rho_{i}, \underset{i \in I}{V} \rho_{i}^{\prime}\right) \in \Theta
$$

In particular, $\Theta$ is a join compatible equivalence relation.

Proof. For each $i \in I$, let $\alpha_{i}$ and $\beta_{i}$ be the least and greatest element respectively of $\rho_{i} \Theta$ and put $\sigma=\bigvee_{i \in I} \alpha_{i}$. Define $H(\sigma)$ as in Section 2. Clearly $H(\sigma)|E=\sigma| E$. Then for each $i \in I$,

$$
B_{i}\left|E=\alpha_{i}\right| E \subseteq \sigma|E=H(\sigma)| E,
$$

and from Theorem $I, \quad \beta_{i} \subseteq H(\sigma)$. Hence $\underset{i \in I}{V} \beta_{i} \subseteq H(\sigma)$. Therefore

$$
\left(\bigvee_{i \in I} \alpha_{i}\right)\left|E \subseteq\left(\bigvee_{i \in I} \beta_{i}\right)\right| E \subseteq H(\sigma)|E=\sigma| E=\left(\bigvee_{i \in I} \alpha_{i}\right) \mid E,
$$

whence $\left(\underset{i \in I}{V} \alpha_{i}\right)\left|E=\left(\underset{i \in I}{\vee} \beta_{i}\right)\right| E$. Since, for each $i \in I$, we have $\alpha_{i} \subseteq \rho_{i} \subseteq \beta_{i}$ and $\alpha_{i} \subseteq \rho_{i}^{\prime} \subseteq \beta_{i}$ we easily obtain that $\left(\underset{i \in I}{V} \rho_{i}\right)\left|E=\left(\underset{i \in I}{V} \rho_{i}^{\prime}\right)^{\prime}\right| E=\left(\underset{i \in I}{V} a_{i}\right) \mid E$, giving the required result.

Following Reilly and Scheiblich [5], we see that

$$
\begin{aligned}
\left(\bigcap_{i \in I} \rho_{i}\right] \cap(E \times E)=\bigcap_{i \in I}\left[\rho_{i} \cap(E \times E)\right] & =\bigcap_{i \in I}\left[\rho_{i}^{\prime} \cap(E \times E)\right] \\
& =\left(\prod_{i \in I} \rho_{i}^{\prime}\right) \cap(E \times E) .
\end{aligned}
$$

From this and Lemma 1 , it follows that $\theta$ is a congruence on $\Lambda(S)$ satisfying condition (A) of Result 1 . The proof of the main theorem is now complete.

COROLLARY 1. For each element $\rho$ in $\Lambda(S)$, let $M(\rho)$ be the greatest element of the $\theta$-class $\rho \Theta$. Then for any two congruences $\rho, \sigma$ on $S$, if $\rho \subseteq \sigma$ then $M(\rho) \subseteq M(\sigma)$.

Proof. Now from Lemma $l, M(\rho) \vee M(\sigma) \in(\rho \vee \sigma) \theta$, and so $M(\rho) \subseteq M(\rho) \vee M(\sigma) \subseteq M(\rho \vee \sigma)=M(\sigma)$.

REMARK 1 . If $m(\rho)$ denotes the least element of $\rho \theta$, then it is clear that $\rho \subseteq \sigma$ implies $m(\rho) \subseteq m(\sigma)$, since $m(\rho)=[\rho \cap(E \times E)]^{*}$, the congruence on $S$ generated by the relation $\rho \cap(E \times E)$. 
REMARK 2. For any $\rho \in \Lambda(S)$, the $\theta$-class $\rho \theta$ is isomorphic to the lattice of idempotent separating congruences on $S / m(\rho)$ (see the proof of (ii) Theorem $3.4[5]$ ) and if $S$ is an inverse semigroup then $\Lambda(S) / \theta$ is (isomorphic to) a sublattice of the lattice of congruences on the semilattice $E$; for let $\rho, \sigma$ be any elements of $\Lambda(S)$. Then $(\rho \cap \sigma) \mid E=(\rho \mid E) \cap(\sigma \mid E)$ and $(\rho \vee \sigma) \mid E=(\rho \mid E) \vee(\sigma \mid E)$ (the proof of Theorem 5.1 [5] shows that $\left.\left(\rho_{1} \vee \rho_{3}\right) \mid E=\left(\rho_{1} \mid E\right) \vee\left(\rho_{3} \mid E\right)\right)$. Let us call $\{\rho \cap(E \times E): \rho \in \Lambda(S)\}$ the set of normal congruences on $E$, and denote it by $N(E)$. Then $N(E)$ is a sublattice of the lattice of congruences on $E$ and is isomorphic to $\Lambda(S) / \Theta$. By considering any Brandt semigroup with three or more idempotents, we see that $N(E)$ is not always equal to the lattice of all congruences on $E$. We note that the normal congruences on $E$ are characterized as follows (due to Reilly and Scheiblich; see Definition 4.1 [5]): a congruence $\zeta$ on $E$ is normal if and only if for every pair $(e, f) \in \zeta$ and every element $a \in S$, we have $\left(a e a^{-1}, a f a^{-1}\right) \in \zeta$.

\section{References}

[1] A.H. Clifford and G.B. Preston, The algebraic theory of semigroups, vols. 1 and 2, (Amer. Math. Soc. Mathematical Surveys 7 , Providence, 1961 and 1967).

[2] G. Lallement, "Congruences et équivalences de Green sur un demi-groupe régulier", C.R. Acad. Sc. Paris, Série A, 262 (1966), 613-616.

[3] Gérard Lallement, "Demi-groupes réguliers" (Doctoral dissertation), Ann. Mat. Pura Appl. (IV), Vol. 77, (1967), 47-130.

[4] W.D. Munn, "A certain sublattice of the lattice of congruences on a regular semigroup", Proc. Cambridge Philos. Soc. 60 (1964), 385-391.

[5] N.R. Reilly and H.E. Scheiblich, "Congruences on regular semigroups", Pacific J. Math. 23 (1967), 349-360.

Department of Mathematics, Monash University, clayton, Victoria. 